\title{
Ambient Mass Spectrometry Imaging: A Comparison of Desorption Ionization by Sonic Spray and Electrospray
}

\author{
Christian Janfelt, ${ }^{1}$ Asger W. Nørgaard ${ }^{2}$ \\ ${ }^{1}$ Department of Pharmacy, University of Copenhagen, Universitetsparken 2, 2100 Copenhagen, Denmark \\ ${ }^{2}$ The National Research Centre for the Working Environment, Lersø Parkalle 105, 2100 Copenhagen, Denmark
}

\begin{abstract}
Easy ambient sonic spray ionization (EASI) and desorption electrospray ionization (DESI) were used for imaging of a number of samples, including sections of rat brain and imprints of plant material on porous Teflon. A novel approach termed Displaced Dual-mode Imaging was utilized for the direct comparison of the two methods: Images were recorded with the individual rows alternating between EASI and DESI, yielding a separate image for each technique recorded under perfectly similar conditions on the same sample. EASI works reliably for imaging of all samples, but the choice of spray solvent and flow rate is more critical in tissue imaging with EASI than with DESI. The overall sensitivity of EASI is, in general, slightly lower than that of DESI, and the representation of the dynamic range is different in images of the two techniques for some samples. However, for abundant compounds, EASI works well, resulting in images of similar quality as DESI. EASI can thus be used in imaging experiments where the application of high voltage is impractical or undesirable. The present study is in its nature also a comparison of the characteristics of the two techniques, showing results also applicable for non-imaging work, with regards to sensitivity and experimental conditions.
\end{abstract}

Key words: Easy ambient sonic spray ionization, Desorption electrospray ionization, Imaging mass spectrometry, Ambient mass spectrometry, Displaced dual-mode imaging

\section{Introduction}

$\mathrm{W}$ ith the introduction of desorption electrospray ionization (DESI) in 2004 and direct analysis in real time (DART) in 2005, it became possible to perform massspectrometric analysis directly from surfaces and under ambient conditions $[1,2]$. The possible applications of the techniques range from direct analysis of tablets $[3,4]$ and profiling of tissues [5] to detection of explosives [6,7] and analysis of liquid samples or extracts deposited on surfaces

Electronic supplementary material The online version of this article (doi:10.1007/s13361-012-0452-5) contains supplementary material, which is available to authorized users.

Correspondence to: Christian Janfelt; e-mail: cja@farma.ku.dk
$[8,9]$, benefiting from the rapid detection offered by these direct techniques.

Since 2004, a variety of related techniques have been invented and applied for many different purposes [10-12]. As for DESI, several of the techniques are desorption analogues of already established spray techniques (e.g., desorption atmospheric pressure chemical ionization (DAPCI) [6], desorption atmospheric pressure photo ionization (DAPPI) [13], and nanospray DESI [14, 15], inspired by APCI, APPI, and nanospray, respectively.

Sonic spray ionization (SSI) [16] is an alternative to electrospray ionization, using no high voltage and thus relying solely on the pressure of the nebulizer gas to induce ionization. Charged droplets and, consequently, gas-phase ions are formed because of an unbalanced charge distribution during droplet formation in the supersonic spray. 
Compared to ESI, SSI results in a softer ionization of (e.g., amino acids) since the ions have lower internal energies than those produced in ESI $[17,18]$. The desorption analogue of SSI was presented in 2006 by Haddad and co-workers, first under the name desorption sonic spray ionization (DeSSI) [19] and later renamed to easy ambient sonic-spray ionization (EASI) [20]. EASI is one of the simplest of the ambient ionization techniques and can be used with any mass spectrometer with an atmospheric pressure inlet. It does not require high voltage or radiation sources and uses only compressed nitrogen and a spray solvent for ionization.

EASI has proven to be efficient in desorbing and ionizing compounds from different types of surfaces, such as drug tablets [19], thin layer chromatographic (TLC) plates [21-23], and paper [24-27]. EASI has been utilized to analyze drugs trapped inside the pores of a molecularly imprinted polymer [28] and for real-time studies of the polymerization of organofunctionalized silanes [29] and degradation of drugs [30]. A combination of DESI-imaging and non-imaging EASI has been suggested for very specific recognition of counterfeit banknotes [31]. Recently, EASI was used for lipid profiling of liver tissue, however, only as liver extracts deposited on a paper surface [23] rather than direct analysis of the liver tissue as previously presented with DESI [5].

DESI imaging has since its introduction in 2006 [32] been used mainly for tissue analysis, (e.g., in imaging of drugs in tissue [33] and examinations of diseased tissue [3436], but also for imaging of plant material [37, 38], studies of metabolite exchange between bacteria [39], defence mechanisms of seaweed [40], and analysis of inks [41] and chemical fingerprints [42].

EASI has not been applied for either direct tissue analysis or imaging. In addition, EASI and DESI have never been compared directly on the same samples. Thus, the scope of this study is to explore and compare these two related techniques in a number of imaging experiments of various samples. Imaging experiments are well suited for the comparison of ionization techniques, since the generation of an image inherently provides a pixel-to-pixel comparison, making it easy to distinguish between signal and noise. It is striking how little signal from a peak is actually needed to provide a good image. Even small peaks that would normally be dismissed as noise may in the comparison with regions in the image, where the compound is not present, indeed turn out to originate from an actual analyte. Therefore, in comparisons of sensitivity, which is all about signal-to-noise ratios, imaging experiments are well suited, although they of course do not provide any quantitation or limits of detection.

\section{Experimental}

\section{Chemicals}

LC-MS grade acetonitrile was purchased from VWR International (Herlev, Denmark) and water was prepared with a Millipore Direct-Q3 UV system (Billerica, MA, USA). All standard compounds were purchased from SigmaAldrich Denmark A/S (Copenhagen, Denmark). Porous Teflon $(1.5 \mathrm{~mm}$ thick, medium pore size of $7 \mu \mathrm{m}$, pore volume of $36 \%$ ) was purchased from Berghof (Eningen, Germany).

\section{Tissue Sections}

The brain was removed from a 9-week old male SpragueDawley rat (Taconic, Ry, Denmark), immediately put on dry ice for rapid freezing, and subsequently placed in a $-80{ }^{\circ} \mathrm{C}$ freezer. The frozen brain was mounted on a cryotome sample holder under $-25{ }^{\circ} \mathrm{C}$ with water as the only adhesive. The brain was cut in slices of $14 \mu \mathrm{m}$ thickness using a Leica CM3050S cryotome (Leica Microsystems, Wetzlar, Germany). Subsequently, the slices were thaw-mounted on glass slides (four sections per glass slide) and stored at $-80{ }^{\circ} \mathrm{C}$ until time of analysis. On the day of analysis, the sample slide was taken directly from the freezer to a vacuum dessiccator for 10 min prior to DESI analysis.

\section{Imprints of Plant Material}

Plants of St. John's Wort (Hypericum perforatum) were harvested in Denmark in September 2011. Imprints were made by pressing the leaves in a sandwich of porous Teflon, leaf, tissue paper, and silicone rubber ( $1 \mathrm{~mm}$ thick) between two aluminum plates in a vice, as described in further details elsewhere [38].

\section{Instrumentation}

Mass spectrometric data were recorded using a Thermo Fisher Scientific LTQ XL Linear Ion Trap Mass Spectrometer (San Jose, CA, USA) equipped with a custom-built DESI imaging ion source. The DESI imaging ion source, described in detail elsewhere [38], was based on a motorized microscope stage from Märzhäuser Wetzlar (Wetzlar, Germany) and controlled by an in-house written software. The mass spectra were collected with Xcalibur 2.0 software (Thermo Fisher Scientific) and converted to image files (imzML files) using a conversion tool (www.maldi-msi.org). The images were generated with Data Cube Explorer from AMOLF, Amsterdam, The Netherlands.

The MS parameters were as follows for negative ion mode experiments: $300{ }^{\circ} \mathrm{C}$ heated capillary temperature, $5 \mathrm{kV}$ spray voltage for DESI experiments $(0 \mathrm{kV}$ for EASI experiments), $-35 \mathrm{~V}$ capillary voltage, $-110 \mathrm{~V}$ tube lens voltage, and automatic gain control (AGC) off. In positive ion mode the capillary voltage was $30 \mathrm{~V}$, the tube lens voltage was $120 \mathrm{~V}$, and the spray voltage was $+5 \mathrm{kV}$ for DESI and $0 \mathrm{~V}$ for EASI.

For the imaging of rat brain (negative ion mode: scan range $m / z 500-1,000$, positive ion mode: scan range $\mathrm{m} / \mathrm{z}$ $600-1,000)$, the geometric parameters were optimized for 
EASI, using a nebulizer gas pressure of 10 bar and a $10 \mu \mathrm{L} /$ min flow of methanol. The ion injection time was $100 \mathrm{~ms}$, and four microscans were averaged for each pixel in the image. A spatial resolution of $150 \mu \mathrm{m}$ was used. The DESI images were recorded under the same conditions, only with $\pm 5 \mathrm{kV}$ high voltage applied to the ion source. Imaging of the plant imprint (negative ion mode, scan range $\mathrm{m} / \mathrm{z} 300-700$ ) was performed with a nebulizer gas pressure of $10 \mathrm{bar}$ and a $2 \mu \mathrm{L} / \mathrm{min}$ flow of acetonitrile and water (50:50). The injection time was $50 \mathrm{~ms}$ with six microscans, and the spatial resolution was $100 \mu \mathrm{m}$. All images were recorded in a displaced dual-mode imaging approach, as discussed below, such that EASI and DESI images were recorded simultaneously of the same sample. The geometry optimization was always performed for EASI and used for both ionization modes.

The nebulizer gas pressure was tested in the range of 515 bar. A major improvement in the EASI signal was observed up to 10 bar, above which no further improvement was observed. For convenience and to avoid unnecessary smearing of the sample, the nebulizer gas pressure was kept at $10 \mathrm{bar}$ in all experiments reported in this paper.

\section{Results and Discussion}

\section{Considerations on Experimental Conditions}

While DESI imaging is typically performed at solvent flow rates of $1-2 \mu \mathrm{L} / \mathrm{min}$ in order to avoid smearing of the sample leading to degraded spatial resolution, EASI analysis is usually performed a higher flow rates (up to $20 \mu \mathrm{L} / \mathrm{min}$ ). DESI is typically performed using mixtures of acetonitrile (or methanol) and water as spray solvent, while mixtures of methanol and water or often pure methanol has been used for EASI.

EASI imaging was possible using a low solvent flow rate, but for tissue samples the geometry optimization was difficult and we were not able to perform any imaging in positive ion mode. However, we found that the optimum way of optimizing the spray geometry for tissue imaging with DESI was to turn off the high voltage and thus optimize in EASI mode. Since the spray geometry is very critical in low flow EASI, the optimal geometry settings could be recognized because of sudden and distinct changes in signal. When the optimal EASI geometry was found, the spray voltage was turned back on, resulting in optimal settings for DESI. By increasing the flow rate of the spray solvent ( $\geq 5 \mu \mathrm{L} / \mathrm{min}$ ) more intense and reliable signals were obtained for EASI. Aqueous solvent mixtures at these high flow rates smear the tissue samples, making imaging impossible. However, by using pure methanol as spray solvent, solvent flows up to $20 \mu \mathrm{L} / \mathrm{min}$ were possible without damaging the sample. A flow of $10 \mu \mathrm{L} / \mathrm{min}$ of pure methanol was found to be ideal for imaging of tissue sections in EASI as well as in DESI. With this, in an imaging context unusually high flow rate, the geometry optimization became very simple for both
EASI and DESI, providing straightforward and reproducible EASI images of brain tissue in negative as well as in positive ion mode.

The sensitivities of EASI and DESI were tested by analyzing standard solutions deposited on a porous Teflon surface, using a $2 \mu \mathrm{L} / \mathrm{min}$ flow of acetonitrile and water (50:50) and a $10 \mu \mathrm{L} / \mathrm{min}$ flow of methanol, respectively. Although $10 \mu \mathrm{L} / \mathrm{min}$ of pure methanol is optimal for EASI analysis of tissue samples, a $2 \mu \mathrm{L} / \mathrm{min}$ flow of the acetonitrile/water mixture was equally good or better for analysis of the standards on Teflon. DESI and EASI showed almost similar sensitivities for compounds, which are easily ionized such as methadone (tens of pg for both techniques). However, for compounds that are more difficult to ionize (e.g., paracetamol) the difference between the two techniques is greater in favor of DESI. The details and results of these experiments are found in the Supplementary Material.

\section{Displaced Dual-Mode Imaging}

In order to make a direct comparison of the two ionization techniques, we designed an experiment where the imaging stage was scanning the sample with for example $100 \mu \mathrm{m}$ spatial resolution in the $x$-direction but in lanes separated by a $50 \mu \mathrm{m}$ distance. Every second lane was scanned in EASI and DESI, respectively, thus generating one EASI image and one DESI image, both with $100 \mu \mathrm{m}$ resolution in both directions. In this way, the same sample was imaged with both techniques, at the same time, and under the same conditions, thus enabling the best possible comparison of the two techniques. In experiments with aqueous (highly conductive) solvents, an equilibration time of $10 \mathrm{~s}$ between each row was sufficient, whereas $50 \mathrm{~s}$ were needed between each row for equilibration of the spray when pure methanol (weakly conductive) was used as spray solvent. We term this approach displaced dual-mode imaging (DDI) and foresee a number of other potential applications of it, as discussed below.

\section{Imaging of Rat Brain Tissue}

EASI and DESI images were recorded in negative and positive ion mode of sections of a rat brain by DDI. Negative ion mode mass spectra extracted from the two images are found in Figure 1, showing EASI and DESI spectra of gray matter areas of the brain (Figure $1 \mathrm{a}$ and $\mathrm{b}$ ) as well as of white matter areas (Figure 1c and d). The general observation is that the spectra, in terms of relative intensities, are similar, showing all the same peaks at quite similar mutual abundances. The only major difference seems to be that the absolute ion intensities are about two times higher for DESI than for EASI. In corresponding spectra recorded with a spray of $2 \mu \mathrm{L} / \mathrm{min} \mathrm{ACN} /$ water (70:30), the DESI intensities are about four times higher than the EASI intensities (Figure S1). It is likely that the impact of the high voltage is less significant in the experiment with pure 
(a)

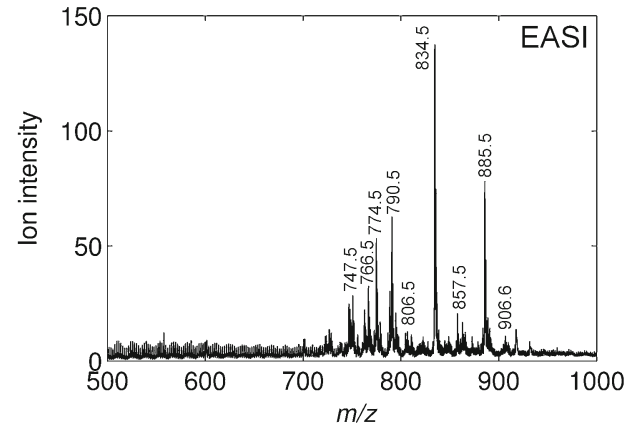

(c)

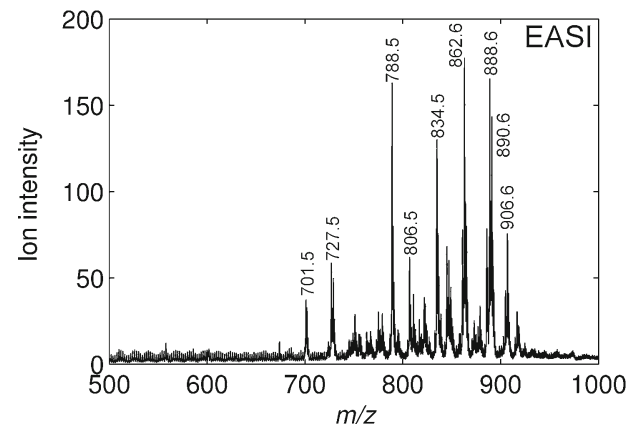

(b)

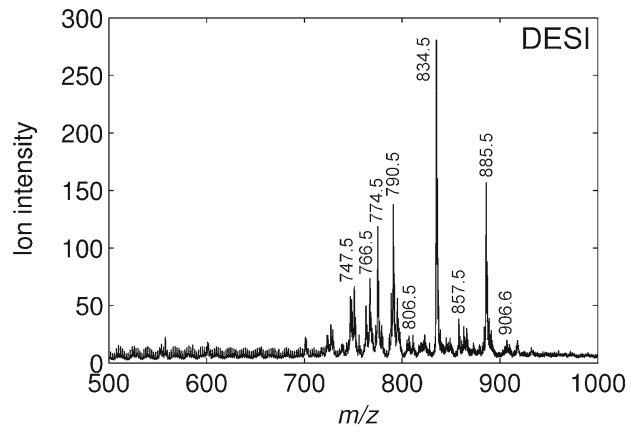

(d)

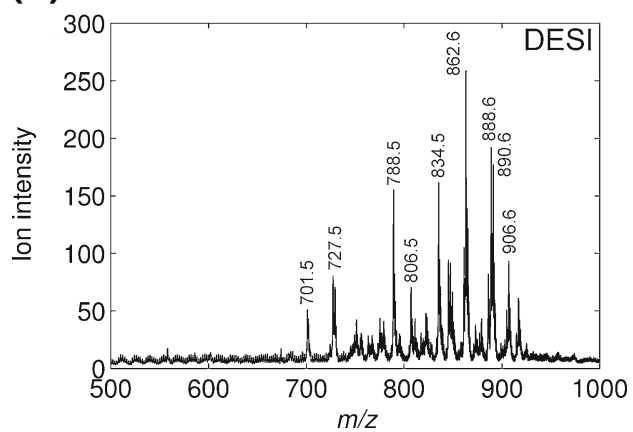

Figure 1. Desorption spectra of sections of rat brain recorded in negative ion mode. The upper row, (a), (b) are of the gray matter, and the lower row, (c), (d) are of the white matter. The left column, (a), (c) are recorded with EASI, and the right column, (b), (d) are recorded with DESI. All spectra are extracted from the images in Figure 2

methanol as spray solvent because of the low conductivity of methanol. The differences between the two techniques become more apparent when images showing the distributions of three different types of lipids are compared (Figure 2). The images differ in the way they show relative intensities in the spatial dimensions. The two lipids PS(40:6) and PI(18:0/20:4) appear in the DESI images (Figure $2 b$ and d) to be present in significantly higher abundances in the gray matter than in the white matter, while the EASI images (Figure $2 \mathrm{a}$ and $\mathrm{c}$ ) show the lipids in more comparable abundances in the two regions. For the sulfatide ST(24:2) the picture seems opposite with DESI showing the lipid present in particular in the white matter but also in parts of the gray matter (Figure 2f), while EASI shows a weaker abundance of the lipid in the gray matter areas (Figure 2e). These differences, which are also observed in images made with a $2 \mu \mathrm{L} / \mathrm{min}$ flow of a 70:30 mixture of acetonitrile/ water (Figure S2), are most likely due to the combined effect of the different dynamic ranges of the techniques (as discussed below) and their respective vulnerabilities with regards to ion suppression. The less intense signal of ST $(24: 2)$ in the gray matter areas in the EASI image in Figure 2e could be ascribed to a greater degree of ion suppression from the highly abundant phospholipids in the gray matter, due to the fewer charges available in an EASI experiment.

It is worth noticing that the spatial resolution of the two techniques is identical, also when compared with images obtained with a low flow of acetonitrile/water (Figure S2). The high flow of methanol does not cause any smearing of the sample.

The positive ion images in Figure 3 show similar effects: the EASI images do not seem to show the same dynamic range as the DESI images. The difference between the white and gray matter areas is observed, but not quite as clearly as in the DESI images. Mass spectra of brain tissue recorded in positive ion mode are found in the Supplementary Material.

\section{Imaging of Plant Imprints}

In another comparison of the two techniques, we performed imaging of Teflon imprints of leaves of Hypericum perforatum, a sample known from a previous study [38]. This sample is ideal for this experiment because the porous Teflon surface is well defined and easy to optimize on, and the plant — studied in hundreds of papers - contains a vast number of different compounds.

Figure $4 \mathrm{c}$ and $\mathrm{d}$ show the EASI and DESI images of hyperforin $(\mathrm{m} / \mathrm{z} 535.4)$ in a leaf of $H$. perforatum. The two images are quite similar, in terms of mutual intensities and details, spatial resolution etc. Figure 5 shows spectra averaged over a complete line in each of the images. As in Figure 1 we see significantly higher absolute intensities (factor of 10) with DESI than with EASI. When zooming in on the two spectra (Figure $5 \mathrm{c}$ and d), it becomes apparent 

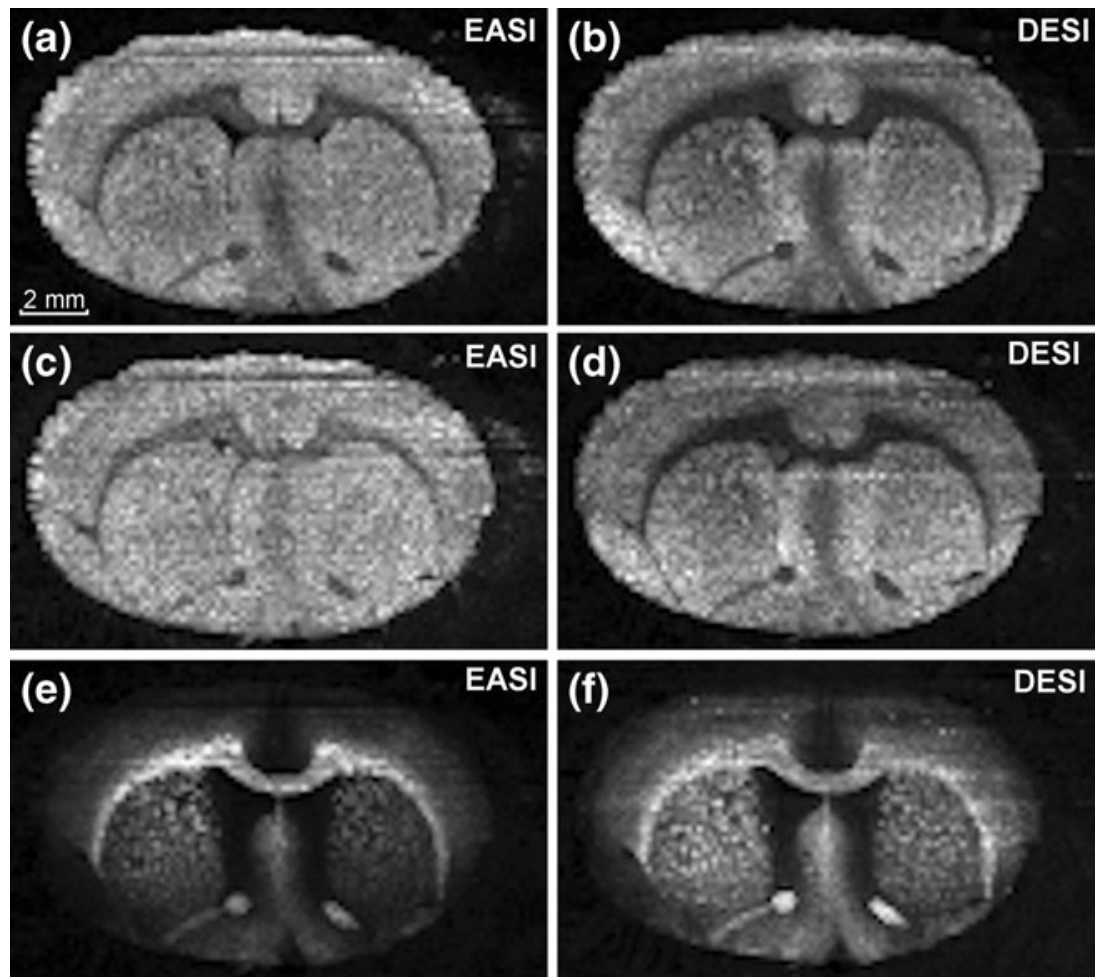

Figure 2. Desorption ionization images of rat brain, recorded with $150 \mu \mathrm{m}$ spatial resolution in negative ion mode. The left column, (a), (c), (e) are recorded with EASI, and the right column, (b), (d), (f) are recorded with DESI. (a), (b): PS(40:6) $(\mathrm{m} / z$ 834.5); (c), (d): $\mathrm{Pl}(18: 0 / 20: 4)(\mathrm{m} / z$ 885.5); (e), (f): ST(24:2) $(\mathrm{m} / \mathrm{z} 888.6)$
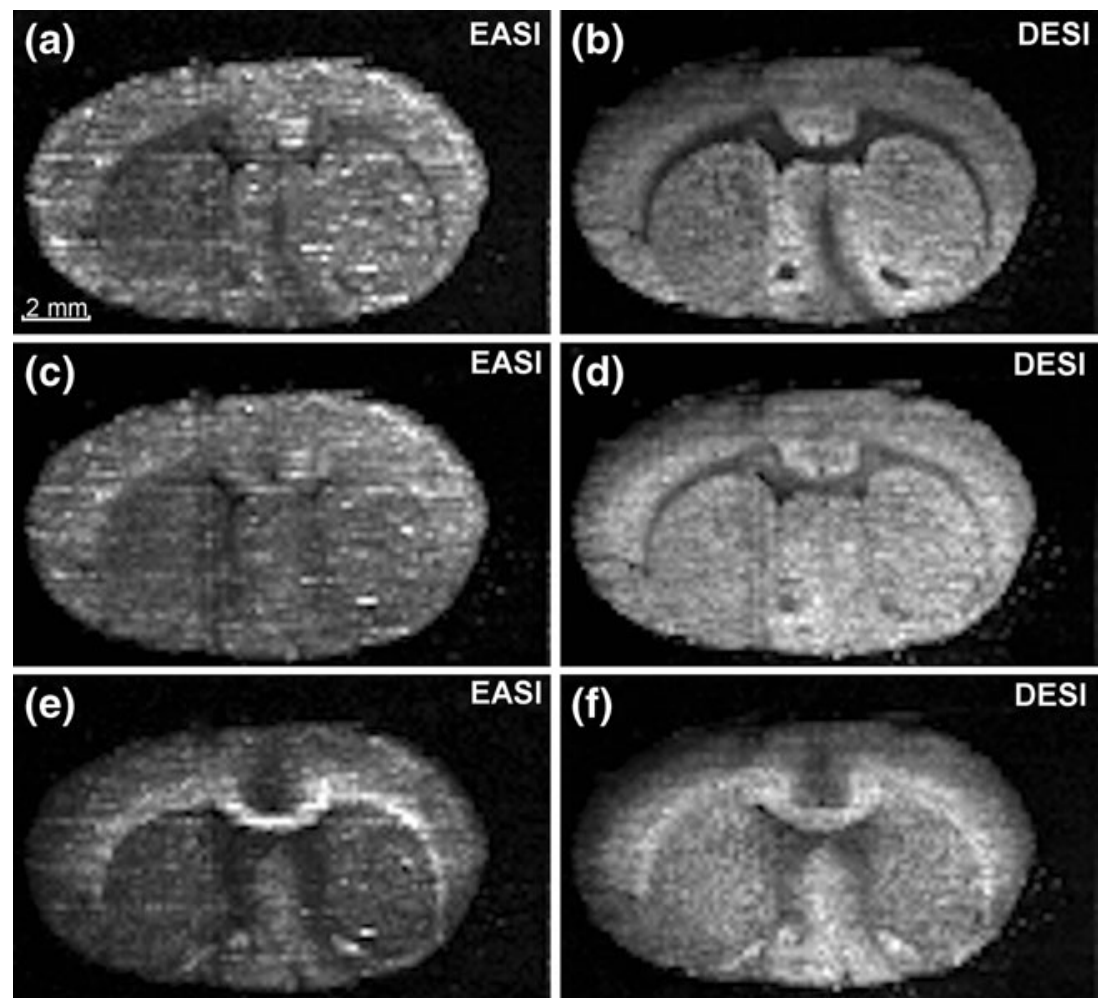

Figure 3. Desorption ionization images of rat brain, recorded with $150 \mu \mathrm{m}$ spatial resolution in positive ion mode (ionized as $\mathrm{K}^{+}$ adducts). The left column, (a), (c), (e) are recorded with EASI, and the right column, (b), (d), (f) are recorded with DESI. (a), (b): PC (32:0) ( $m / z$ 772.5); (c), (d): PC(34:1) $(m / z$ 798.5); (e), (f): PC(36:1) $(\mathrm{m} / z$ 826.6) 

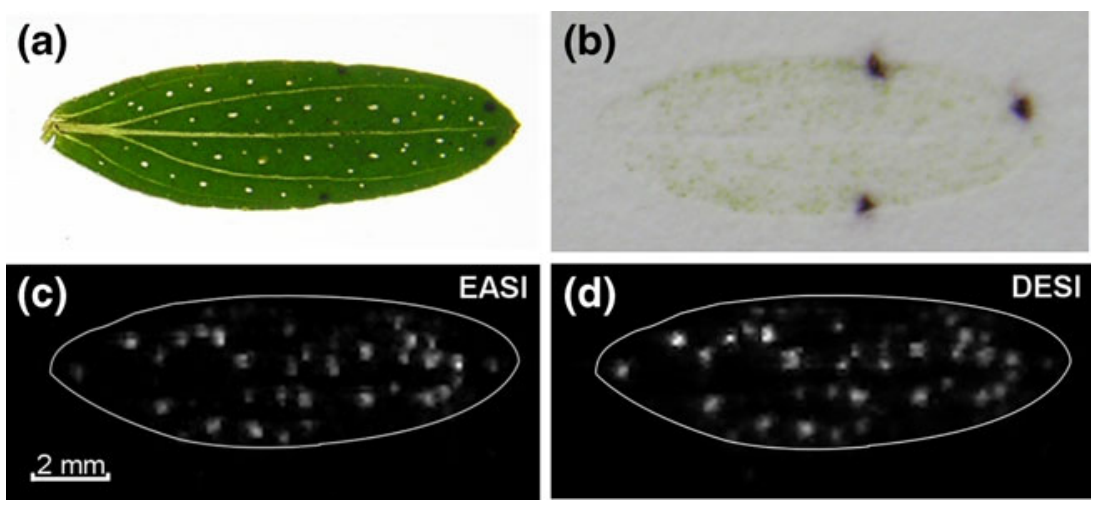

Figure 4. Leaf of Hypericum perforatum. (a) Leaf ; (b) imprint; (c) image of hyperforin ( $\mathrm{m} / \mathrm{z} 535.4)$ recorded by EASI; (d) image of hyperforin $(m / z 535.4)$ recorded by DESI

that more of the low-abundance compounds are observed with DESI than with EASI.

The difference in the dynamic range between EASI and DESI noticed during the imaging of rat brain is also apparent in the images of the plant imprint. Figure $6 \mathrm{a}$ and $\mathrm{b}$ show the EASI and DESI images of hypericin $(\mathrm{m} / \mathrm{z} 503.1)$, respectively. Hypericin is highly abundant in black glands on the leaf and is therefore transferred in high amounts to the porous Teflon surface during the imprinting process. The EASI image (Figure 6a) shows three spots of hypericin that appear to be stretching in lines from the spots toward the lower right corner of the image. These stretched shapes clearly indicate the movement of the sprayer over the surface (relative movement, as the sprayer is indeed kept still while the surface is moving). As the sprayer moves in a line from left to right and the lines are scanned one by one, starting from the top and moving towards the bottom, apparently the sprayer can, because of the spray solvent and the pressure from the nebulizer gas, move to some extent tiny amount of the sample in the bottom-right direction. This effect is not observed in the corresponding DESI image of the very same surface, and neither were there any visual indications on the (a)

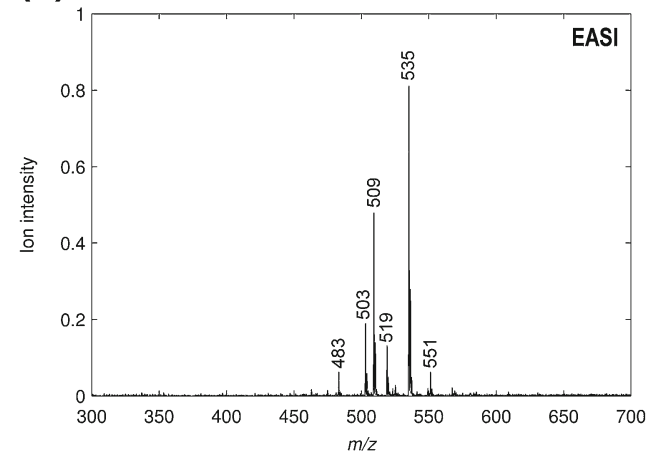

(c)

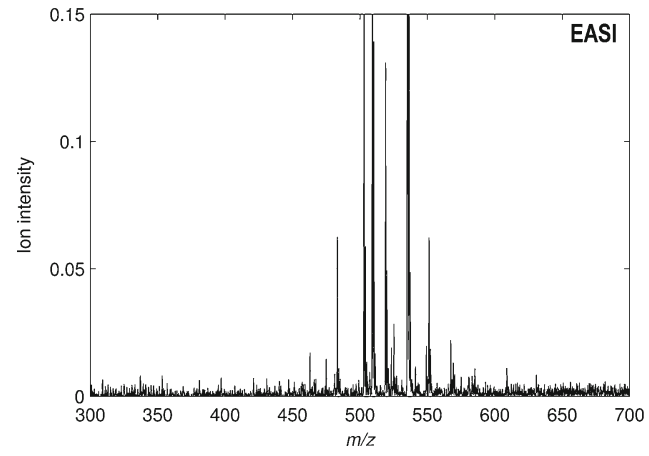

(b)

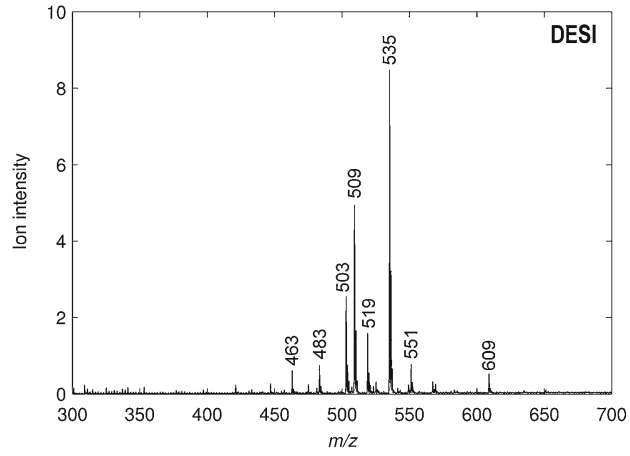

(d)

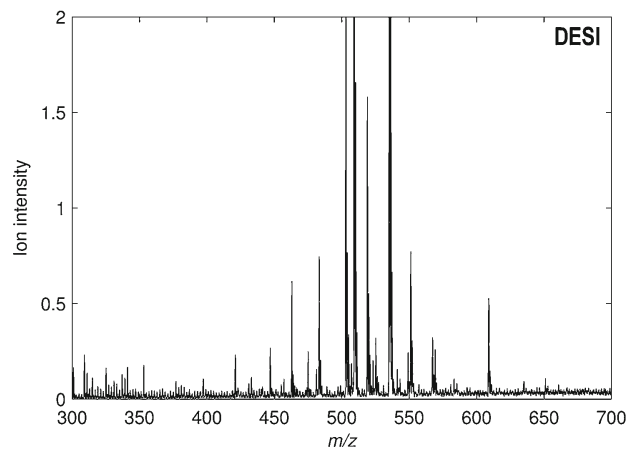

Figure 5. Desorption spectra of a leaf of Hypericum perforatum, averaged over one full line from the images in Figure 4. The left column, (a), (c) are recorded with EASI, and the right column, (b), (d) are recorded with DESI; (c), (d) are the same spectra as (a), (b), but zoomed on the ordinate axis in order to show the signal to noise ratios of the weakest signals 

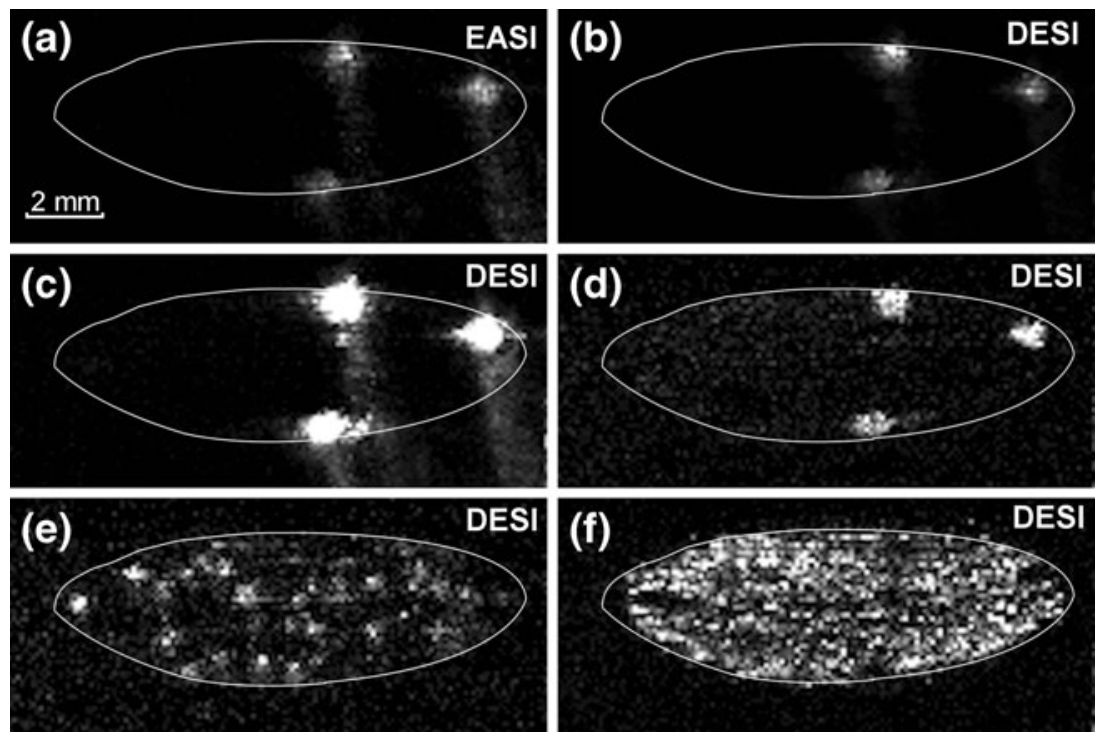

Figure 6. Desorption ionization images of a leaf of Hypericum perforatum. (a) EASI image of hypericin ( $\mathrm{m} / \mathrm{z} 503.1)$, normalized such that a white pixel corresponds to an intensity of 12 (arb. units). (b) DESI image of hypericin $(\mathrm{m} / z$ 503.1), normalized such that a white pixel corresponds to an intensity of 200 (arb. units). (c) DESI image of hypericin $(\mathrm{m} / z$ 503.1), normalized such that a white pixel corresponds to an intensity of 20 (arb. units). (d) DESI image of quercetin ( $\mathrm{m} / z$ 301.0). (e) DESI image of an oxidation product of hyperforin $(\mathrm{m} / \mathrm{z}$ 583.4). (f) DESI image of rutin $(\mathrm{m} / \mathrm{z}$ 609.2)

surface that the black material on the surface had been moved in such lines. It turned out, however, that the lines were also recorded in the DESI images, although only at levels so low that they were not visible in the normal color scaling of the image. If the color scale is changed in order to "zoom in" on lower signal intensities, he lines become visible. This is in practice done by defining which absolute intensity is required to produce a white pixel in the images, and all intensities below this threshold are then properly scaled in shades of gray such that an intensity of 0 results in a black pixel. Typically, the highest signal in the image of the ion being studied is used as threshold. For example, the EASI image in Figure 6a was generated such that an absolute intensity of 12 (arb. units) equals a white pixel, while the DESI image in Figure 6b with its higher intensities was generated with 200 as the threshold. If this threshold in the DESI image is lowered to 20 (i.e., a factor of 10 lower than the highest signal in the image), the hypericin lines show up in the DESI image as well (Figure 6c). This shows that the lines in the EASI images are not an indication that EASI is more sensitive than DESI- the lines are detected with both techniques - but that EASI has a somewhat lower dynamic range than DESI. Although the amount of sample transported by the sprayer is most likely orders of magnitude lower than in the original hypericin spots (no visual signs of any transport), the EASI image portrays the concentrations as being of similar order of magnitude.

The higher sensitivity of DESI observed in the spectra in Figure 5 is also expressed in the images of various compounds from the $H$. perforatum plant from the EASI and DESI experiments. As seen in Figure 6, DESI was capable of imaging several low abundance compounds.
From the DESI data we could generate images of quercetin $(\mathrm{m} / \mathrm{z}$ 301.0, Figure 6d) (known to accumulate in the black glands and therefore have the same distribution as hypericin [43]), an oxidation product of hyperforin $(\mathrm{m} / \mathrm{z} 583.4$, Figure 6e) [44] (which therefore has the same distribution as hyperforin), and rutin ( $m / z$ 609.2, Figure 6f), which is homogeneously distributed throughout the leaf surface and also previously shown to be found in the $H$. perforatum leaves [45]. None of these compounds showed up in images generated by EASI.

\section{Imaging of Dyes on Paper and PMMA in Positive Ion Mode}

EASI has previously been used for analysis of inks and dyes, and was therefore expected to be suitable for imaging of such samples. Thus, letters written using red and blue permanent markers on paper and PMMA were used for testing of the imaging performance of EASI in the positive ion mode. The red pen provides a signal in positive ion mode at $\mathrm{m} / \mathrm{z} 443$, corresponding to Rhodamine $6 \mathrm{G}$, and the blue pen provides a signal in positive ion mode at $\mathrm{m} / \mathrm{z} 478$, corresponding to Basic Blue 7, as well as a minor signal at $\mathrm{m} / \mathrm{z} 443$ due to Rhodamine B. The two isomeric rhodamines can be distinguished by MS/MS: Rhodamine $6 \mathrm{G}$ yields a major fragment at $\mathrm{m} / \mathrm{z} 415$, while the major fragment of Rhodamine B is $m / z$ 399. It should be noted that all three dyes are cationic in their natural state, typically occurring as chloride salts and thus easily ionized. The critical point is therefore extraction and desorption of these compounds from the surface. 
The imaging performances of EASI and DESI of the three dyes on paper and PMMA were equally good and with comparable absolute signal intensities. EASI appears to be quite tolerant with respect to contaminations (e.g., in the solvent line); the ion of $\mathrm{m} / \mathrm{z} 443$ is always the base peak, even on contaminated systems. In comparison, DESI is more vulnerable to contamination and shows several peaks in the background on a contaminated system. In EASI with its lower number of charges available, there seems to be a more pronounced preference for donating the limited amount of charges to the compound that is the most easily ionized, in this case the cationic dyes. In case of the blue dye, the $\mathrm{m} / \mathrm{z}$ 443 peak (Rhodamine B) is slightly less prevalent, relative to Basic Blue 7 at $m / z 478$, in the EASI spectra than in the DESI spectra. The spatial resolution of the two techniques is similar, providing very comparable images down to a resolution of about $100 \mu \mathrm{m}$. The experimental details and results of these experiments are found in the Supplementary Material.

\section{Conclusions}

We have shown that EASI can be used for imaging as well as direct analysis of tissue sections. For analytes such as dyes, the performance is similar to DESI, even presenting a larger tolerance of contaminants in the analytical system. Imaging of tissue samples in negative and positive ion modes was possible although optimization of the solvent composition and flow rate is more crucial in EASI than with DESI. A $10 \mu \mathrm{L} / \mathrm{min}$ flow of methanol seems to be optimal, providing images with very high success ratios. These solvent settings also work well for DESI imaging of tissue, demanding very little in terms of geometry optimization in both positive and negative ion mode. The need for a higher solvent flow rate in EASI is probably due to the lower number of charges on the droplets, which is then compensated for by generation a larger number of droplets via an increased flow rate. The EASI images turned out a little differently than the corresponding DESI images, indicating differences in the dynamic range or some degree of ion suppression. However, EASI is well-suited for imaging of a variety of samples and analytes, in particular when the analyte is of relatively high abundance or easily ionized. EASI imaging can thus prove useful in imaging applications where high voltage (DESI) is impractical or undesirable (e.g., in potential imaging of living organisms).

In conclusion, we have introduced displaced dual-mode imaging, a new method to record two images with different ionization settings of the very same sample simultaneously, by using half the pixel size in the $y$-direction as in the $x$ directly and change the ionization settings for every second row in the image. We used it here to make a reliable estimation of the impact of the high voltage on the sensitivity in desorption imaging experiment by generating simultaneous images with and without high voltage. This enabled a direct comparison of the sensitivity and perfor- mance of EASI and DESI, which is otherwise difficult to obtain because of difficulties in reproducing geometries, samples, and spray conditions. A more general application, however, would be use it as a method to record simultaneous imaging in positive and negative ion modes of the same sample. This would be a good extension of the recent work by Cooks and co-workers, where one sample was used for consecutive DESI imaging, MALDI imaging, and H\&E staining [46].

\section{Acknowledgment}

The authors thank Niels Wellner for providing the rat brain and Janina Thunig for useful discussions about compounds in Hypericum perforatum. Support from the Carlsberg Foundation, The Danish Council for Independent Research | Natural Sciences and the Working Environment Research Fund, Denmark, is gratefully acknowledged.

\section{References}

1. Takats, Z., Wiseman, J.M., Gologan, B., Cooks, R.G.: Mass spectrometry sampling under ambient conditions with desorption electrospray ionization. Science 306, 471-473 (2004)

2. Cody, R.B., Laramee, J.A., Durst, H.D.: Versatile new ion source for the analysis of materials in open air under ambient conditions. Anal. Chem. 77, 2297-2302 (2005)

3. Samms, W.C., Jiang, Y.J., Dixon, M.D., Houck, S.S., Mozayani, A.: Analysis of Alprazolam by DART-TOF mass spectrometry in counterfeit and routine drug identification cases. J. Forensic Sci. 56, 993-998 (2011)

4. Nyadong, L., Late, S., Green, M.D., Banga, A., Fernández, F.M.: Direct quantitation of active ingredients in solid artesunate antimalarials by noncovalent complex forming reactive desorption electrospray ionization mass spectrometry. J. Am. Soc. Mass Spectrom. 19, 380-388 (2008)

5. Wiseman, J.M., Puolitaival, S.M., Takats, Z., Cooks, R.G., Caprioli, R.M.: Mass spectrometric profiling of intact biological tissue by using desorption electrospray ionization. Angew. Chem. Int. Ed. 44, 70947097 (2005)

6. Takats, Z., Cotte-Rodriguez, I., Talaty, N., Chen, H., Cooks, R.G.: Direct, trace level detection of explosives on ambient surfaces by desorption electrospray ionization mass spectrometry. Chem. Commun. 1950-1952 (2005)

7. Nilles, J.M., Connell, T.R., Stokes, S.T., Dupont Durst, H.: Explosives detection using direct analysis in real time (DART) mass spectrometry. Propellants Explos. Pyrotech. 35, 446-451 (2010)

8. Kauppila, T.J., Talaty, N., Kuuranne, T., Kotiaho, T., Kostiainen, R., Cooks, R.G.: Rapid analysis of metabolites and drugs of abuse from urine samples by desorption electrospray ionization-mass spectrometry. Analyst 132, 868-875 (2007)

9. Thunig, J., Flø, L., Pedersen-Bjergaard, S., Hansen, S.H., Janfelt, C.: Liquid-phase microextraction and desorption electrospray ionization mass spectrometry for identification and quantification of basic drugs in human urine. Rapid Commun. Mass Spectrom. 26, 133-140 (2012)

10. Chen, H., Gamez, G., Zenobi, R.: What can we learn from ambient ionization techniques? J. Am. Soc. Mass Spectrom. 20, 1947-1963 (2009)

11. Alberici, R., Simas, R., Sanvido, G., Romão, W., Lalli, P., Benassi, M., Cunha, I., Eberlin, M.: Ambient mass spectrometry: bringing MS into the "real world." Anal. Bioanal. Chem. 398 (2010)

12. Harris, G.A., Nyadong, L., Fernandez, F.M.: Recent developments in ambient ionization techniques for analytical mass spectrometry. Analyst 133, 1297-1301 (2008)

13. Haapala, M., Pól, J., Saarela, V., Arvola, V., Kotiaho, T., Ketola, R.A., Franssila, S., Kauppila, T.J., Kostiainen, R.: Desorption atmospheric pressure photoionization. Anal. Chem. 79, 7867-7872 (2007) 
14. Roach, P.J., Laskin, J., Laskin, A.: Nanospray desorption electrospray ionization: an ambient method for liquid-extraction surface sampling in mass spectrometry. Analyst 135, 2233-2236 (2010)

15. Laskin, J., Heath, B.S., Roach, P.J., Cazares, L., Semmes, O.J.: Tissue imaging using nanospray desorption electrospray ionization mass spectrometry. Anal. Chem. 84, 141-148 (2011)

16. Hirabayashi, A., Sakairi, M., Koizumi, H.: Sonic spray mass spectrometry. Anal. Chem. 67, 2878-2882 (1995)

17. Takats, Z., Nanita, S.C., Cooks, R.G., Schlosser, G., Vekey, K.: Amino acid clusters formed by sonic spray ionization. Anal. Chem. 75, 15141523 (2003)

18. Sørensen, M.B., Aaslo, P., Egsgaard, H., Lund, T.: Determination of D/ L-amino acids by zero needle voltage electrospray ionisation. Rapid Commun. Mass Spectrom. 22, 455-461 (2008)

19. Haddad, R., Sparrapan, R., Eberlin, M.N.: Desorption sonic spray ionization for (high) voltage-free ambient mass spectrometry. Rapid Commun. Mass Spectrom. 20, 2901-2905 (2006)

20. Haddad, R., Sparrapan, R., Kotiaho, T., Eberlin, M.N.: Easy ambient sonic-spray ionization-membrane interface mass spectrometry for direct analysis of solution constituents. Anal. Chem. 80, 898-903 (2008)

21. Haddad, R., Milagre, H.M.S., Catharino, R.R., Eberlin, M.N.: Easy ambient sonic-spray ionization mass spectrometry combined with thinlayer chromatography. Anal. Chem. 80, 2744-2750 (2008)

22. Eberlin, L.S., Abdelnur, P.V., Passero, A., de Sa, G.F., Daroda, R.J., de Souza, V., Eberlin, M.N.: Analysis of biodiesel and biodieselpetrodiesel blends by high performance thin layer chromatography combined with easy ambient sonic-spray ionization mass spectrometry. Analyst 134, 1652-1657 (2009)

23. Alberici, L., Oliveira, H., Catharino, R., Vercesi, A., Eberlin, M. Alberici, R.: Distinct hepatic lipid profile of hypertriglyceridemic mice determined by easy ambient sonic-spray ionization mass spectrometry. Anal. Bioanal. Chem. 401, 1655-1663 (2011)

24. Haddad, R., Catharino, R.R., Marques, L.A., Eberlin, M.N.: Perfume fingerprinting by easy ambient sonic-spray ionization mass spectrometry: nearly instantaneous typification and counterfeit detection. Rapid Commun. Mass Spectrom. 22, 3662-3666 (2008)

25. Abdelnur, P.V., Eberlin, L.S., de Sá, G.F., de Souza, V., Eberlin, M.N.: Single-shot biodiesel analysis: nearly instantaneous typification and quality control solely by ambient mass spectrometry. Anal. Chem. 80, $7882-7886$ (2008)

26. Simas, R.C., Catharino, R.R., Cunha, I.B.S., Cabral, E.C., BarreraArellano, D., Eberlin, M.N., Alberici, R.M.: Instantaneous characterization of vegetable oils via TAG and FFA profiles by easy ambient sonic-spray ionization mass spectrometry. Analyst 135, 738-744 (2010)

27. Lalli, P.M., Sanvido, G.B., Garcia, J.S., Haddad, R., Cosso, R.G., Maia, D.R.J., Zacca, J.J., Maldaner, A.O., Eberlin, M.N.: Fingerprinting and aging of ink by easy ambient sonic-spray ionization mass spectrometry. Analyst 135, 745-750 (2010)

28. Figueiredo, E.C., Sanvido, G.B., Zezzi Arruda, M.A., Eberlin, M.N. Molecularly imprinted polymers as analyte sequesters and selective surfaces for easy ambient sonic-spray ionization. Analyst 135, 726-730 (2010)

29. Nørgaard, A.W., Vaz, B.G., Lauritsen, F.R., Eberlin, M.N.: Real-time monitoring of the progress of polymerization reactions directly on surfaces at open atmosphere by ambient mass spectrometry. Rapid Commun. Mass Spectrom. 24, 3441-3446 (2010)

30. Amaral, P.H., Fernandes, R., Eberlin, M.N., Höehr, N.F.: Direct monitoring of drug degradation by easy ambient sonic-spray ionization mass spectrometry: the case of enalapril. J. Mass Spectrom. 46, 1269$1273(2011)$

31. Eberlin, L.S., Haddad, R., Sarabia Neto, R.C., Cosso, R.G., Maia, D.R.J., Maldaner, A.O., Zacca, J.J., Sanvido, G.B., Romao, W., Vaz,
B.G., Ifa, D.R., Dill, A., Cooks, R.G., Eberlin, M.N.: Instantaneous chemical profiles of banknotes by ambient mass spectrometry. Analyst 135, 2533-2539 (2010)

32. Wiseman, J.M., Ifa, D.R., Song, Q.Y., Cooks, R.G.: Tissue imaging at atmospheric pressure using desorption electrospray ionization (DESI) mass spectrometry. Angew. Chem. Int. Ed. 45, 7188-7192 (2006)

33. Kertesz, V., Van Berkel, G.J., Vavrek, M., Koeplinger, K.A., Schneider, B.B., Covey, T.R.: Comparison of drug distribution images from whole-body thin tissue sections obtained using desorption electrospray ionization tandem mass spectrometry and autoradiography. Anal. Chem. 80, 5168-5177 (2008)

34. Eberlin, L.S., Dill, A.L., Costa, A.B., Ifa, D.R., Cheng, L., Masterson, T., Koch, M., Ratliff, T.L., Cooks, R.G.: Cholesterol sulfate imaging in human prostate cancer tissue by desorption electrospray ionization mass spectrometry. Anal. Chem. 82, 3430-3434 (2010)

35. Masterson, T., Dill, A., Eberlin, L., Mattarozzi, M., Cheng, L., Beck, S., Bianchi, F., Cooks, R.: Distinctive glycerophospholipid profiles of human seminoma and adjacent normal tissues by desorption electrospray ionization imaging mass spectrometry. J. Am. Soc. Mass Spectrom. 22, 1326-1333 (2011)

36. Janfelt, C., Wellner, N., Leger, P.-L., Kokesch-Himmelreich, J., Hansen, S.H., Charriaut-Marlangue, C., Hansen, H.S.: Visualization by mass spectrometry of 2-dimensional changes in rat brain lipids, including $\mathrm{N}$-acylphosphatidylethanolamines, during neonatal brain ischemia. FASEB J. 26, 2667-2673 (2012)

37. Li, B., Bjarnholt, N., Hansen, S.H., Janfelt, C.: Characterization of barley leaf tissue using direct and indirect desorption electrospray ionization imaging mass spectrometry. J. Mass Spectrom. 46, 1241$1246(2011)$

38. Thunig, J., Hansen, S.H., Janfelt, C.: Analysis of secondary plant metabolites by indirect desorption electrospray ionization imaging mass spectrometry. Anal. Chem. 83, 3256-3259 (2011)

39. Watrous, J., Hendricks, N., Meehan, M., Dorrestein, P.C.: Capturing bacterial metabolic exchange using thin film desorption electrospray ionization-imaging mass spectrometry. Anal. Chem. 82, 1598-1600 (2010)

40. Lane, A.L., Nyadong, L., Galhena, A.S., Shearer, T.L., Stout, E.P., Parry, R.M., Kwasnik, M., Wang, M.D., Hay, M.E., Fernandez, F.M., Kubanek, J.: Desorption electrospray ionization mass spectrometry reveals surface-mediated antifungal chemical defense of a tropical seaweed. Proc. Natl. Acad. Sci. U. S. A. 106, 7314-7319 (2009)

41. Ifa, D.R., Gumaelius, L.M., Eberlin, L.S., Manicke, N.E., Cooks, R.G.: Forensic analysis of inks by imaging desorption electrospray ionization (DESI) mass spectrometry. Analyst 132, 461-467 (2007)

42. Ifa, D.R., Manicke, N.E., Dill, A.L., Cooks, G.: Latent fingerprint chemical imaging by mass spectrometry. Science 321, 805 (2008)

43. Holscher, D., Shroff, R., Knop, K., Gottschaldt, M., Crecelius, A., Schneider, B., Heckel, D.G., Schubert, U.S., Svatos, A.: Matrix-free UV-laser desorption/ionization (LDI) mass spectrometric imaging at the single-cell level: distribution of secondary metabolites of Arabidopsis thaliana and Hypericum species. Plant J. 60, 907-918 (2009)

44. Verotta, L., Appendino, G., Belloro, E., Jakupovic, J., Bombardelli, E.: Furohyperforin, a Prenylated Phloroglucinol from St. John's Wort (Hypericum perforatum). J. Nat. Prod. 62, 770-772 (1999)

45. Dogrukol-Ak, D., Kirimer, N., Tunçel, M., Aboul-Enein, H.Y.: Determination of rutin in Hypericum perforatum extract by capillary electrophoresis. Anal. Lett. 34, 185-191 (2001)

46. Eberlin, L.S., Liu, X., Ferreira, C.R., Santagata, S., Agar, N.Y.R., Cooks, R.G.: Desorption electrospray ionization then MALDI mass spectrometry imaging of lipid and protein distributions in single tissue sections. Anal. Chem. 83, 8366-8371 (2011) 